\title{
Adaptation of radar software to work with ambiguous distance measurement
}

\section{Kamil Stawiarski, Michał Meller}

Kamil Stawiarski, Michał Meller, "Adaptation of radar software to work with ambiguous distance measurement," Proc. SPIE 11442, Radioelectronic Systems Conference 2019, 1144205 (11 February 2020); doi: $10.1117 / 12.2565118$ 


\title{
Adaptation of radar software to work with ambiguous distance measurement
}

\author{
Kamil Stawiarski ${ }^{\mathrm{a}, \mathrm{b}}$ and Michał Meller ${ }^{\mathrm{a}, \mathrm{b}}$ \\ aPIT-RADWAR S.A., ul. Poligonowa 30, 04-051 Warsaw, POLAND \\ ${ }^{\mathrm{b}}$ Gdańsk University of Technology,, Faculty of Electronics, Telecommunications and Computer \\ Science, Department of Automatic Control, ul. Narutowicza 11/12, 80-233 Gdańsk, POLAND
}

\begin{abstract}
A software extension for radar stations designed to work in the low-PRF mode that allows them to correctly measure range to targets outside of their instrumented range, is proposed. The solution does not require substantial modifications of the radar software. Additionally, we describe tools that allow one to simulate the output of a low-PRF radar observing targets that are outside its instrumented range. The proposed approach is verified using computer simulations.
\end{abstract}

Keywords: Radiolocation, ambiguous distance measurement, estimation

\section{INTRODUCTION}

Distance to the target is one of the basic parameters determined by radar. It is desirable to have as wide range as possible, however, it is usually associated with increasing time between two scans of the target. In effect coordinates are updated less frequently. It happens because of the longer waiting time for receiving the transmitted signal. The compromise between both parameters is selected based on the purpose of the given radar. Fast information refresh is crucial for weapon locating radars $\left(\mathrm{WLR}^{12}\right)$. Short pulse repetition period is also the cornerstone of the mode which allows to recognize hovering helicopter. ${ }^{3}$ In this case, high pulses frequency is desirable to build frequency statistics of the received signal, which requires a significant number of observations. On the opposite side it can be placed radars whose quick access to information is not crucial, so they can have a much greater range, reaching up to $3000 \mathrm{~km}$ (in the case of over-the-horizon radars ${ }^{4}$ ). They often form part of a larger early warning system. Significant ranges are also required in airport surveillance radar (ASR).

The short range problem of radars with short pulse repetition period can be alleviated by using ambiguous distance measurements. Using such a procedure requires that the impulses in subsequent space scans be slightly shifted in time with respect to each other. Adding additional technical times results in a longer pulse repetition period, but it remains incomparably smaller than in the case of obtaining a similar range without using an ambiguous distance measurement.

For normal operation, the pulse radar has two basic antenna modes. At first signal is transmitted, reaches the target and reflects from it. ${ }^{5}$ At the end it reaches the radar, in which the antenna is in the meantime switched to receive mode. Distance of the target can be calculated based on the signal propagation time. The choice of transmission time determines the dead zone (longer pulse, longer dead zone, but also more of radiated energy). The observation time, in turn, translates into the maximum detection range (a longer waiting for receiving the reflected signal indicates that it has traveled a longer distance). In pulse radars, the transmission / receiving sequences follow in succession, allowing the completion of several target illuminations, which makes detection of the target more efficient. During work with ambiguous distance measure, target can be detected despite the fact of being significantly above the basic range of the radar. The signal reflected from the target reaches the antenna in furthers receiving sequences than than it would appear in normal working mode. In effect detection appears at the basic range but echo has reflected from the target at a much further distance. For a constant pulse

Further author information: (Send correspondence to Kamil Stawiarski)

Kamil Stawiarski: E-mail: kamil.stawiarski@pg.edu.pl

Radioelectronic Systems Conference 2019, edited by Piotr Kaniewski, Jan Matuszewski, Proc. of SPIE

Vol. 11442, 1144205 - (c) 2020 SPIE · CCC code: 0277-786X/20/\$21 · doi: 10.1117/12.2565118 
repetition period, this distance will be always the same in subsequent repetition periods, however, adding delays according to previous scans will cause appearing of detection at different distances in each segment. Appropriate recognition and transformation of those detection distances allows to obtain the actual distance of the target.

The paper considers the problem of unraveling the correct distance of the target during work with ambiguous distance measurement mode. The main difficulty is to recognize the sequence of detections that will indicate the presence of the target above the basic range of the radar and transforming them in the way which allows to obtain the real distance of the observed object. The proposed algorithm is based on the division of the problem into several parts, which in their entirety constitute a complete set of tools to solve it. The first part describes how to transform a sequence of known pulse for obtaining detection in basic range based on hypothetical target placed at distance beyond that range. The second one is the reverse task - basing on hypothetical detection in the basic range allows to specify possible out-of-range target placements which could cause detection at given distance. The last part of the algorithm consists in appropriate sequence matching that will allow correct classification of the target within the range of ambiguous measurement. An additional assumption was that the algorithm should interfere with the implemented signal processing as little as possible. It has placed immediately after the detection module and operates on the list of elementary detections. The result of its operation is the modification of input data by merging the detection specified as coming from the above of basic radar range and redefining their real distances.

\section{ISSUE OF DETECTION IN DISTANCE ABOVE BASIC RADAR RANGE}

\subsection{Specification of transmitted signal}

It is assumed that the radar illuminates the target with pulses composed of technical time $\left(\mathbf{T}_{d}\right.$, placed at the beginning of the scan, vector of values for several segments), transmission time (duration $\tau_{T x}$ ) and the time of receiving of the transmitted signal (duration $\tau_{R x}$ ). A train of $N$ of such packages is called a segment. Within each segment, the shown times must remain constant for the received signal to remain coherent. This is a requirement for a Doppler filtration. The next segment needs to have technical time different than the preceding one. This is crucial for determining the target distance beyond the primary range. The detection module gives detection based on data from individual segments. To solve the problem of ambiguous distance measurement, it is required to collect data from several (we denote this number as $M$ ) segments.

An increase in this number improves the quality of the ambiguous distance estimate, and in addition reduces the likelihood of false classification. However, it requires a longer signal collection time. When using the algorithm, it is required to create a array of start/end times for transmitting/receiving signals:

$$
\begin{aligned}
\mathbf{T}_{T x S}[s, p]=(s * N+p) *\left(\tau_{R x}+\tau_{T x}\right)+N * \sum_{n=0}^{s-1} \mathbf{T}_{\text {tech }}(n)+(p+1) * \mathbf{T}_{d}(s) \\
\mathbf{T}_{T x E}[s, p]=\mathbf{T}_{T x S}[s, p]+\tau_{T x} \\
\mathbf{T}_{R x S}[s, p]=\mathbf{T}_{T x E}[s, p]+\tau_{T x} \\
\mathbf{T}_{R x E}[s, p]=\mathbf{T}_{T x E}[s, p]+\tau_{R x}
\end{aligned}
$$

where $s$ denotes number of segment, $p$ - pulse number, $\mathbf{T}_{T x}$ and $\mathbf{T}_{R x}$ denotes the array of transmitting/receiving times respectively (depending on additional indexes beginning or ending of a given time). In fact, the antenna goes into receive mode as soon as transmission is completed, then $\mathbf{T}_{R x S}$ should be the same as $\mathbf{T}_{T x E}$ but offset of length $\tau_{T x}$ is caused by the waiting time to receive the full broadcast signal and creates a dead zone. In addition, for each pulses is is defined number of previous transmitting sequences from which data can be received. Theoretically, it is possible to observe all previously given pulses, but in practice it is worth to limit their number to $N_{\text {wrap }}$. The number of transmission pulses observed is determined by the formula:

$$
P_{T x N o}(s, p)=\min \left((s-1) * N+p, N_{\text {wrap }}\right)
$$



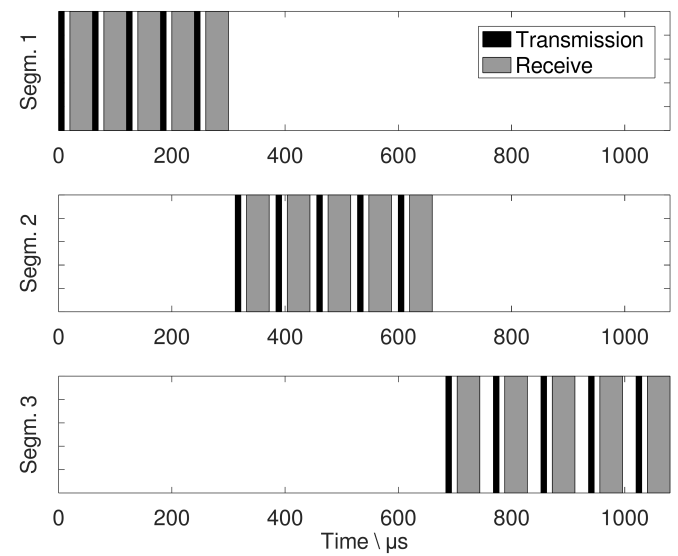

Figure 1. Timeline of transmitting/receiving signals.

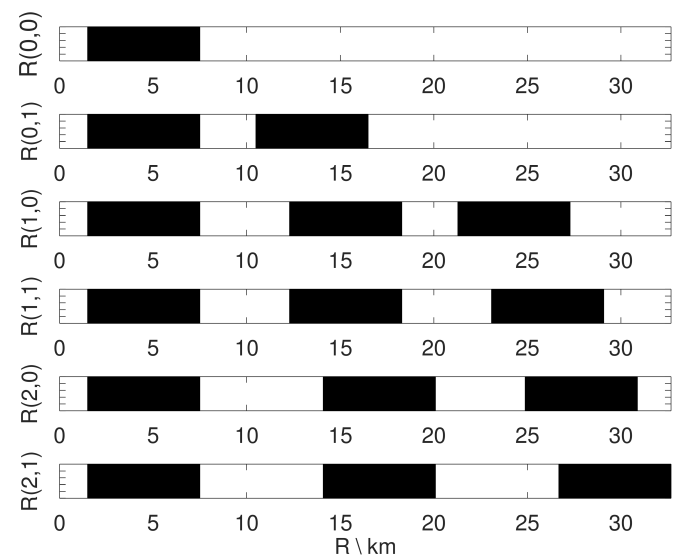

Figure 2. The range of observed distances shown for the first 2 pulses of each of the three segments.

Using the formulas above, for each pulse, it can be defined the distance ranges that their can observe (using the range equation ${ }^{6}$ )

$$
\begin{aligned}
& R_{S}(s, p, r)=\frac{\mathbf{T}_{R x S}[s, p]-\mathbf{T}_{T x E}[s, p-r]}{2 c} \\
& R_{E}(s, p, r)=\frac{\mathbf{T}_{R x E}[s, p]-\mathbf{T}_{T x E}[s, p-r]}{2 c}
\end{aligned}
$$

assuming $\mathbf{T}_{T x E}[s,-r]=\mathbf{T}_{T x E}[s-1, p-r] . r$ denotes number of backward scan, from which the signal is received. Based on the values $\tau_{T x}=10 \mu s, \tau_{R x}=50 \mu s, \mathbf{T}_{\text {tech }}=[0 \mu s, 12 \mu s, 24 \mu s], N=5, M=3, N_{\text {wrap }}=3$ it has been generated plots 1 i 2.

\subsection{Determination of the out-of-range echo detection range}

To determine the distance in range over which a distant target will be detected it can be used formula 8 . It allows to check if the target at the distance $R$ is observed by probing $p$ in the segment $s$ on distance ring $r$. The formula gives the detection distance in the basic range, or 0 if the target is not observable.

$$
\operatorname{Det}_{\text {Range }}(s, p, r, R)=\left\{\begin{array}{l}
R-R_{S}(s, p, r)+R_{S}(s, p, 0) \quad \text { if } R_{S}(s, p, r)<R<R_{E}(s, p, r) \\
0 \text { otherwise }
\end{array}\right.
$$

At this point, an additional function has to be defined. They are a generalization of the formula $8-\operatorname{Det}_{\text {Range }}(R)$. It takes only 1 argument - the distance of a hypothetical object, and returns a vector of distances at which the 
target can be observed using all available scans. This is done by calculating $\operatorname{Det}_{\text {Range }}(s, p, r, R)$ for each available $s, p, r$, and the results are written sequentially to the output vector. In the last step, it is need to review the output vector, leaving only unique values different from zero. To reduce the probability of false detection, the first few probes from each segment can be omitted (ideally $N_{\text {wrap }}$ scans). It comes from the fact that in the first probing of the first segment, observing echo from target at distance above basic range is not possible. In subsequent segments, these detections are shifted in such a way that they appear only in one probe. The chances of detecting such a target are negligible, and taking into account such a detection distance significantly increases the likelihood of incorrect distance estimation. During removing of duplicated detections, it is worth to consider the detection stage (the minimum distance separating two adjacent detections) and remove such elements from the vector that it would remain only those spaced by at least $R_{D i f f M i n}$.

\subsection{Determination of the hypothetical distance based on detection in basic range}

It were used methods similar to those shown in previous section. At first, it needs to be calculated range of ambiguous distances wherein can be found the target, which caused detection on distance $R$ :

$$
\operatorname{Det}_{H y p}(s, p, r, R)=R-R_{S}(s, p, 0)+R_{S}(s, p, r)
$$

The formula 9 works correctly assuming $R_{S}(s, p, 0)<R<R_{E}(s, p, 0)$. Again, it is need to generalize the function $\operatorname{Det}_{H y p}(s, p, r, R)$ to the form $\operatorname{Det}_{H y p}(R)$, which returns vector distances. Each element is a hypothesis of true target placement. In this function removing of duplicated values is also needed (and eventually spaced less than $R_{\text {DiffMin }}$ ). In this case, it is also recommended to complete the vector without the first few probings for reasons analogous to those described in the previous section. Importantly, the vector returned by the function $\operatorname{Det}_{H y p}(s, p, r, R)$ also contains the given distance $R$.

\subsection{Cost function}

The cost function might be understood as function, which takes the hypothetical distance $R$ and vector of detections $\mathbf{R}_{D e t}$, and returns a degree of similarity between them:

$$
J\left(R, \mathbf{R}_{\text {Det }}\right)=\sum_{n=0}^{N_{\mathbf{R}_{\text {Det }}}} \sum_{m=0}^{N_{\mathbf{R}_{\text {Range }}}} \exp \left(-\frac{\left(\mathbf{R}_{\text {Det }}[n]-\mathbf{R}_{\text {Range }}[m]\right)^{2}}{\left(3 * R_{\text {DiffMin }}\right)^{2}}\right)
$$

where $\mathbf{R}_{\text {Range }}=\operatorname{Det}_{\text {Range }}(R)$ is a vector of detections in range for a target at a distance of $R$. The above formula bases on the probability density function of Gaussian distribution. ${ }^{7}$ The value for ideal detection should be equal to the number of detections in the basic range $\left(J\left(R, \operatorname{Det}_{\text {Range }}(R)\right)=\operatorname{dim}\left(\operatorname{Det}_{\text {Range }}(R)\right)\right.$, where $\operatorname{dim}()$ is the number of elements in the vector). The cost function may be larger if the detection vector has a well-matched detection vector and additionally redundant detections located at a similar distance to echoes obtained from the hypothetical target. For example: $J\left(\hat{R},\left[\operatorname{Det}_{\text {Range }}(\hat{R}) \operatorname{Det}_{\text {Range }}\left(\hat{R}+R_{\text {DiffMin }}\right)\right]\right) \sim 5.1496$.

\subsection{Unraveling ambiguities in a distance}

The algorithm for resolving the actual distance of the target above of the basic range requires the use all of formulas and algorithms presented so far. It can be represented as a function $\operatorname{Det}_{A D M}(\mathbf{R})$. As the input data, true detections vector is given. Those data comes from detection module directly $-\mathbf{R}_{D e t}$. For each element of that vector, hypothetical target distances that are beyond the elemental range are taken using $\mathbf{R}_{H y p}=\operatorname{Det}_{H y p}\left(\mathbf{R}_{D e t}\right)$. The next step is to determine which of the hypothetical distances has the largest value of the cost function:

$$
H_{i d x}=\underset{x}{\operatorname{argmax}} J\left(\mathbf{R}_{H y p}[x], \mathbf{R}_{D e t}\right)
$$

Knowing the target distance with the greatest value of the cost function $\left(\mathbf{R}_{H y p}\left[H_{i d x}\right]\right)$, it can be rewritten into complex detection $R_{C p x}$. By complex detection is meant detection extended by vector of distance. Those distances comes from basic detections. In case of proper recognition single complex detections contains all signals coming from single target (a few different ranges in case of ambiguous detection). It is need to obtain vector of detections which could be caused by most likely target: $\mathbf{R}_{\text {Search }}=\operatorname{Det}_{\text {Range }}\left(\mathbf{R}_{H y p}\left[H_{i d x}\right]\right)$. For each element of 


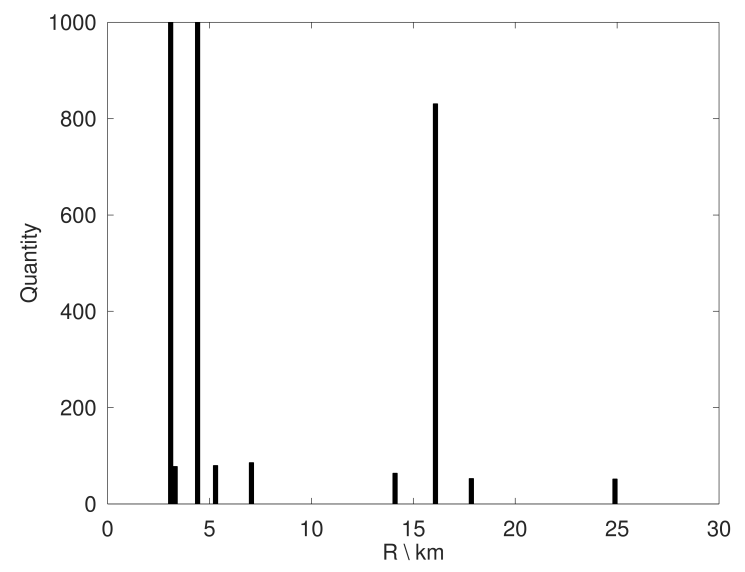

Figure 3. Histogram showing simulation results (white noise distortion).

the vector $\mathbf{R}_{\text {Search }}$ it is calculated square of difference between its distance and distance of each element from list of detections $\mathbf{R}_{D e t}$ in search of a minimum:

$$
H_{\text {Search }}=\underset{x}{\operatorname{argmin}}\left(\mathbf{R}_{\text {Search }}[x]-\mathbf{R}_{\text {Det }}[k]\right)^{2}
$$

If $\left(\mathbf{R}_{\text {Det }}\left[H_{\text {Search }}\right]-\mathbf{R}_{\text {Det }}[k]\right)^{2}<\left(3 * R_{\text {DiffMin }}\right)^{2}$ is fulfilled, then element $\mathbf{R}_{\text {Det }}\left[H_{\text {Search }}\right]$ is removed from the list $\mathbf{R}_{D e t}$ and added as a component in the vector of complex detection at a distance $\mathbf{R}_{H y p}\left[H_{i d x}\right]$. After completing the list of components (searching the minimum for each element of the $\mathbf{R}_{\text {Search }}$ ) it should be looked again at the complex detection. In the case when it contains only one component detection - it is very likely that it comes from target in basic range. In that case distance of complex detection has to be overwritten by distance of its component.

The detection obtained in this way is added to the $\mathbf{R}_{C p x}$ list, and the algorithm continues using the reduced vector $\mathbf{R}_{D e t}$. This procedure is being repeated until detection vector is completely emptied.

\section{SIMULATION RESULTS}

For the purposes of simulation, the following parameters were used: $\tau_{T x}=10 \mu s, \tau_{R x}=50 \mu s, \mathbf{T}_{t e c h}=$ $[0 \mu s, 12 \mu s, 24 \mu s], N=5, M=3, N_{\text {wrap }}=3$.

In that case $\operatorname{Det}_{\text {Range }}(R=15000)=[2408.74207 .56006 .2]$.

By operating the reverse of the above: $\operatorname{Det}_{A D M}([2408.74207 .56006 .2])=14999.97$.

To better show how the algorithm works, the test data vector has been simulated: [3000 $4500 \operatorname{Det}_{\text {Range }}(16000)$ ], which was additionally disturbed by white noise of variance $\sigma^{2}=R_{D i f f M i n}^{2}$. This simulation was made for 1000 tests. The results histogram is presented on the graph 3. It shows that in the case of additional detections from the range $(3000 \mathrm{~m}$ and $4500 \mathrm{~m})$ the recognition efficiency as an echo in the range is $100 \%$. When the echo is in the ambiguous zone (distance $15 \mathrm{~km}$ ), the recognition efficiency is above $80 \%$. In cases of misdiagnosis, the algorithm estimated the distance $\sim 14 \mathrm{~km}, \sim 18 \mathrm{~km}, \sim 25 \mathrm{~km}$, or determined the detection as coming from the basic range $(\sim 3.2 \mathrm{~km}, \sim 5.2 \mathrm{~km}, \sim 7 \mathrm{~km})$. Each situation is about $5 \%$ of cases.

The simulation has been repeated with the same distances, but interfering noise has changed to uniform with variance $\sigma^{2}=0.5 * R_{D i f f M i n}^{2}$. Simulation results are presented in figure 4 . It is clearly seen that every target has been classified correctly.

\section{SUMMARY}

The problem of ambiguous distance measurement in the impulse radar was considered. Tools have been proposed to determine the range of detections based on the target in the area of ambiguous measurement, as well as 


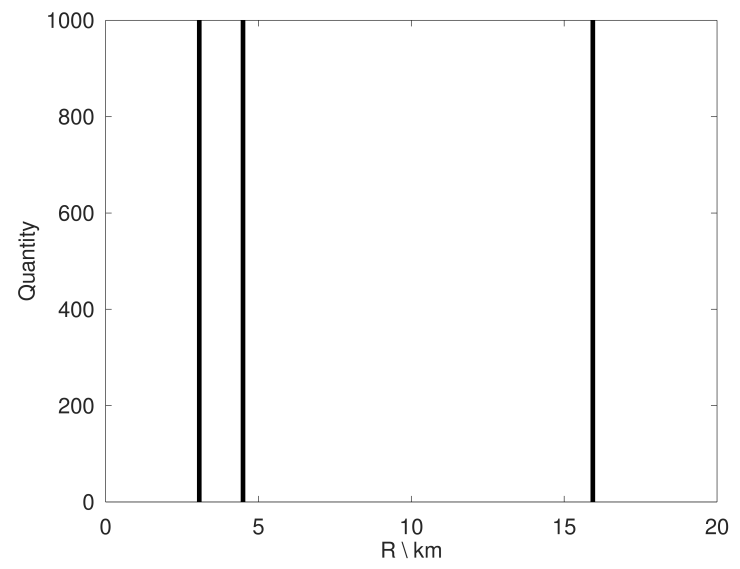

Figure 4. Histogram showing simulation results (uniform noise distortion).

hypothetical detections based on the given detections. The proposed method allows the recognition of a distant target (in the area of ambiguous measurement) and its classification with high accuracy. An additional advantage is the ability to use in existing methods without modifying existing software.

\section{REFERENCES}

[1] Czekała, Z., [Parada radarów], 372-380, Bellona (1999).

[2] "Tactics, techniques, and procedures for field artillery target acquisition." HEADQUARTERS, DEPARTMENT OF THE ARMY (June 2002).

[3] Xiaobin Feng, Jin Yan, Peikang Huang, and Zhihe Xiao, "Detection of hovering helicopter," in [6th International Conference on Signal Processing, 2002.], 2, 1449-1452 vol.2 (2002).

[4] Czekała, Z., [Parada radarów], 363-368, Bellona (1999).

[5] Czekała, Z., [Parada radarów], 15-22, Bellona (1999).

[6] Richards, M. A., [Fundamentals of Radar Signal Processing], 1-6, McGraw-Hill Professional (2005).

[7] Kay, S. M., [Fundamentals of Statistical Signal Processing: Estimation Theory], ch. Maximum Likelihood Estimation, Prentice-Hall, Inc., Upper Saddle River, NJ, USA (1993). 